\title{
Interrogation of O-ATRP Activation Conducted by Singlet and Triplet Excited States of Phenoxazine Photocatalysts
}

Yisrael M. Lattke, Daniel A. Corbin, Steven M. Sartor, Blaine G. McCarthy, Garret M. Miyake, and Niels H. Damrauer

\section{Abstract}

Organocatalyzed ATRP (O-ATRP) is a growing field exploiting organic chromophores as photoredox catalysts (PCS) that engage in dissociative electron transfer (DET) activation of alkyl halide initiators following absorption of light. Characterizing DET rate coefficients $\left(k_{\text {act }}\right)$ and photochemical yields across various reaction conditions and PC photophysical properties will inform catalyst design and efficient use during polymerization. The studies described herein consider a class of phenoxazine PCs where synthetic handles of core-substitution and $\mathrm{N}$-aryl substitution enable tunability of the electronic and spin character of the catalyst excited state as well as DET reaction driving force $\left(\Delta G_{E T}^{0}\right)$. Using Stern-Volmer quenching experiments through variation of diethyl 2-bromo-2-methylmalonate (DBMM) initiator concentration, collisional quenching is observed. Eight independent measurements of $k_{\text {act }}$ are reported as a function of $\Delta G_{E T}^{0}$ for four PCs: four triplet reactants and four singlets with $k_{\text {act }}$ values ranging from $1.1 \times 10^{8}$ $\mathrm{M}^{-1} \mathrm{~s}^{-1}$ where DET itself controls the rate to $4.8 \times 10^{9} \mathrm{M}^{-1} \mathrm{~s}^{-1}$ where diffusion is rate limiting. This overall data set, as well as a second one inclusive of five literature values from related systems, is readily modeled with only a single parameter of reorganization energy under the frameworks of adiabatic Marcus electron transfer theory and Marcus-Savéant theory of DET. The results provide a predictive map where $k_{a c t}$ can be estimated if $\Delta G_{E T}^{0}$ is known and highlight that DET in these systems appears insensitive to PC reactant electronic and spin properties outside of their impact on driving force. Next, on the basis of measured $k_{\text {act }}$ values in selected PC systems and knowledge of their photophysics, we also consider activation yields specific to the reactant spin states as the DBMM initiator concentration is varied. In N-naphthyl-containing PCs characterized by near-unity intersystem crossing, the $T_{1}$ is certainly an important driver for efficient DET. 
However, at DBMM concentrations common to polymer synthesis, the $S_{1}$ is also active and drives $33 \%$ of DET reaction events. Even in systems with low yields of ISC, such as in N-phenyl-containing PCs, reaction yields can be driven to useful values by exploiting the $S_{1}$ under high DBMM concentration conditions. Finally, we have quantified photochemical reaction quantum yields, which take into account potential product loss processes after electron-transfer quenching events. Both $\mathrm{S}_{1}$ and $\mathrm{T}_{1}$ reactant states produce the $\mathrm{PC}^{\circ+}$ radical cation with a common yield of $71 \%$, thus offering no evidence for spin selectivity in deleterious back electron transfer. The subunity $\mathrm{PC}^{\bullet+}$ yields suggest that some combination of solvent (DMAC) oxidation and energy-wasting back electron transfer is likely at play and these pathways should be factored in subsequent mechanistic considerations.

\section{Introduction}

Atom transfer radical polymerization $(A T R P)^{1,2}$ is an established method enabling the generation of polymers with narrow molecular-weight distributions and predictable chain lengths that are useful in demanding industrial ${ }^{3-5}$ and biomedical ${ }^{6-8}$ settings. Polymerization control in ATRP is established through manipulation of two key reaction steps: activation and deactivation. The former occurs when a terminal alkyl-bromide bond on a polymer chain is reductively cleaved by the catalyst to generate a reactive radical on the polymer chain that can grow by propagation. Deactivation is the reverse reaction, occurring when a bromide is reinstalled onto the radical polymer chain, thus stopping further growth until subsequent activation. Because of their importance, there have been efforts to modulate the relative rates of activation and deactivation through thermal, ${ }^{9}$ electrochemical, ${ }^{10}$ sonic, ${ }^{11}$ and optical ${ }^{12,13}$ means.

Although ATRP is a mature materials synthesis methodology, trace contamination from the transition-metal catalysts remains a concern for biomedical and electronic applications, ${ }^{10,14,15}$ resulting in a push for metal-free variants. Organocatalyzed ATRP (O-ATRP) is one such alternative. It exploits organic chromophores as photoredox catalysts (PCs) that engage in activation following absorption of light. Since the inception of O-ATRP using perylene ${ }^{16}$ and $N$ phenyl-phenothiazine, ${ }^{17}$ PC scope has blossomed to include derivatives of phenazine, ${ }^{18,19}$ 
phenoxazine, ${ }^{20-23}$ phenothiazine, ${ }^{24-26}$ acridine, ${ }^{27}$ carbazole, ${ }^{28}$ and thienothiophene. ${ }^{29}$ Several of these PCs have demonstrated the level of control over a polymerization that is expected from traditional ATRP. The use of light to drive a polymerization further provides many exciting opportunities for spatial and temporal control. ${ }^{30,31}$ However, significant additional complexity emerges due to the reliance on photophysical properties and excited-state reactivity. Maturation of this technology requires an understanding of these newly introduced mechanistic details.

The synthetic modularity of many of the organic PC platforms used in O-ATRP has enabled the generation of molecular libraries with diverse properties relevant to photoredox catalysis. ${ }^{32,33}$ This modularity includes energetics for critical redox-driven chemical steps, excited-state lifetimes of participatory states, excited-state electronic character such as spin and/or the degree of charge transfer, and the yield with which reactive states are formed. ${ }^{34}$ However, it remains unclear how these PC properties impact O-ATRP, and specifically how they influence activation and deactivation. In this work, our aim is to contribute to the understanding of how PC properties influence or control activation. We focus on developing a predictive driving-force relationship for this process and address the nature in which both electronic and spin character of excited states is relevant.

In O-ATRP the necessary driving force for the electron transfer tied to activation comes from the PC being in a photoexcited state. A catalytic cycle (Scheme 1) begins with the absorption of visible light by the ground-state singlet ${ }^{1} \mathrm{PC}$ resulting in the formation of an excited singlet state $\left({ }^{1} \mathrm{PC}^{*}\right)$. In competition with its own radiative and nonradiative decay processes, the ${ }^{1} \mathrm{PC}^{*}$ can now either participate directly in activation $\left(k_{a c t, S_{1}}\right)$ or if the rate of intersystem crossing to the triplet state $\left(k_{/ s c}\right)$ is competitive given the polymerization conditions, form a triplet state $\left({ }^{3} \mathrm{PC}^{*}\right)$ that subsequently engages in activation $\left(k_{a c t, T_{1}}\right)$. Like in metal-catalyzed ATRP, activation is the reductive cleavage of a $\mathrm{P}_{\mathrm{n}}-\mathrm{Br}$ bond resulting in an activated radical polymer chain, $\mathrm{P}_{\mathrm{n}}{ }^{\bullet}$, the catalyst radical cation, $\mathrm{PC}^{\bullet+}$, and a bromide ion, $\mathrm{Br}^{-}$. The latter two species are anticipated to exist in equilibrium with the association complex $\left[\mathrm{PC}^{\circ+} \mathrm{Br}^{-}\right] \cdot{ }^{35}$ The rest of the catalytic cycle is comparable to the traditional ATRP mechanism. Namely, the active chain $\mathrm{P}_{\mathrm{n}}{ }^{\bullet}$ grows in length 
( $\left.k_{\text {prop }}\right)$ before it is deactivated by $\left[\mathrm{PC}^{\bullet+} \mathrm{Br}^{-}\right]$, returning the polymer to its dormant state $\left(\mathrm{P}_{\mathrm{n}+\mathrm{m}}-\mathrm{Br}\right)$ and regenerating ${ }^{1} \mathrm{PC}$.

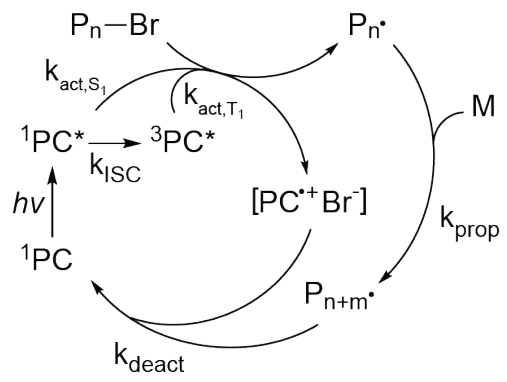

Scheme 1: A proposed O-ATRP catalytic cycle. Both the ${ }^{1} \mathrm{PC}^{*}$ and ${ }^{3} \mathrm{PC} *$ can participate in the activation process, with respective rate coefficients $k_{a c t, S_{1}}$ and $k_{a c t, T_{1}}$. In both cases the products of activation are identical.

Whereas $k_{\text {act }}$ has been extensively explored in traditional ATRP ${ }^{36-38}$, few values have been reported for O-ATRP. Those values that have been reported indicate that $k_{\text {act }}$ can approach values associated with a diffusion-limited process. From these studies $35,39,40$ it has become apparent that there are several unresolved questions about how PC molecular properties impact $k_{\text {act }}$. The first concept involves the overarching question of the relationship between the driving force for electron transfer, $\Delta G_{E T}^{0}$, and the value of $k_{\text {act. }}$ Understanding this relationship would enable prediction of $k_{\text {act }}$ in future systems following basic measurements of ground and excited-state thermodynamic quantities. The second question, which is tied to $\Delta G_{E T}^{0}$, has to do with whether the singlet or triplet excited state of the PC is the dominant reactant under synthetic conditions. Certainly, high-yield long-lived triplet excited states are commonly desired in solution-phase photoredox catalysis ${ }^{41}$ and can be engineered in organic systems without heavy atoms by exploiting molecular substructures with orthogonal $\pi$ orbitals to increase spin-orbit coupling and promote intersystem crossing. ${ }^{42-46}$ However, the predecessor singlet excited states often have lifetimes on the order of ones to tens of nanoseconds, which can be long enough to engage in bimolecular photochemistry as long as there is sufficient concentration of the reactive partner. The third question involves intramolecular charge-transfer (CT) excited states and the observation that PCs possessing such states perform better in O-ATRP by the metrics of polymer dispersity and initiator efficiency. Because of this observation, there has been discussion ${ }^{39,47}$ regarding the relevance or necessity of CT states for expediting forward electron transfer due to 
a greater degree of coupling for the electron transfer process. The current work will consider $k_{\text {act }}$ from the lens of these three questions.

\section{Results and Discussion}

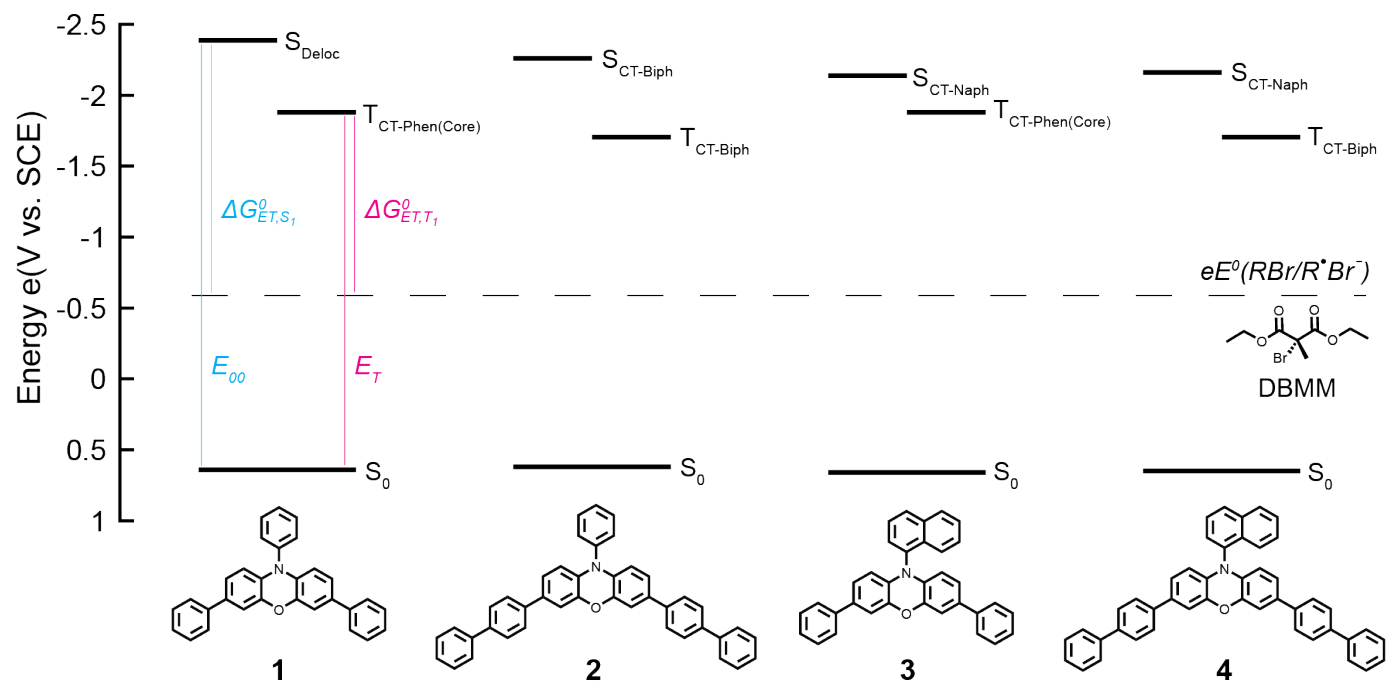

Figure 1: Molecules (PCS 1-4 and DBMM) investigated in this study and the energy levels of their ground and excited states, expressed on a scale that sets the zero of energy at the energy corresponding to a one-electron reduction requiring $0 \mathrm{~V}$ vs. SCE. Greater negative values on this scale represent greater reducing power. The $\mathrm{S}_{0}$ energy levels correspond to the reduction $\left(\mathrm{PC}^{\bullet+} / \mathrm{PC}\right)$, whereas the $\mathrm{S}_{1}$ and $\mathrm{T}_{1}$ energy levels are calculated using Eq. 3 and a modified form of it (Eq. S6), respectively (see SI for details and reduction potential values). The dashed line is the energy corresponding to the potential at which DBMM is reduced. The blue and pink lines highlight the quantities used in Eq. 4 for calculating $\Delta G_{E T}^{0}$. The small (-47 meV) Coloumbic contribution is omitted from the figure.

Table 1: Free energies for the ET reaction from $\mathrm{S}_{1}$ and $\mathrm{T}_{1}$ states of PCs 1-4 and associated $k_{\text {act }}$ values

\begin{tabular}{|c|c|c|c|c|c|c|c|}
\hline PC & $\tau_{0 . S_{1}}(\mathrm{~ns})$ & $\Delta G_{E T, S_{1}}^{0}(\mathrm{eV})$ & $k_{a c t, S_{1}}\left(\times 10^{9} \mathrm{M}^{-1} \mathrm{~s}^{-1}\right)^{a}$ & $\Phi_{I S C}$ & $\tau_{0, T_{1}}(\mathrm{~ms})$ & $\Delta G_{E T, T_{1}}^{0}(\mathrm{eV})$ & $k_{a c t, T_{1}}\left(\times 10^{9} \mathrm{M}^{-1} \mathrm{~s}^{-1}\right)^{b}$ \\
\hline 1 & 3.24 & -1.87 & $4.80 \pm 0.09$ & $0.30^{c}$ & 1.5 & -1.34 & $0.19 \pm 0.01$ \\
\hline 2 & 2.87 & -1.72 & $3.3 \pm 0.3$ & $0.11^{d}$ & 1.2 & -1.18 & $0.120 \pm 0.009$ \\
\hline 3 & 6.32 & -1.59 & $2.0 \pm 0.1$ & $0.95^{c}$ & 1.5 & -1.34 & $0.20 \pm 0.01$ \\
\hline 4 & 5.2 & -1.62 & $2.0 \pm 0.1$ & $0.91^{d}$ & 0.48 & -1.16 & $0.110 \pm 0.009$ \\
\hline
\end{tabular}

${ }^{a}$ Reported error is twice the standard deviation of a triplicate five-point dataset. ${ }^{b}$ Reported error comes from the regression analysis of a single ten-point dataset (Fig. 3). ${ }^{C}$ Values from our previous work. ${ }^{42}$ Values from our previous work. ${ }^{43} \mathrm{~A}$ detailed table including quantities needed for the calculation of $\Delta G_{E T}^{0}$ is provided in Table S1.

Photophysical Background. The four phenoxazine PCs 1-4 (Figure 1; Table 1) studied herein were selected for their collective variety in triplet yields $\left(\Phi_{I S C}\right), \Delta G_{E T}^{0}$, and degree of excited-state CT character, which will allow us to address each of the three questions mentioned above. Their study yielded eight experimental values of $k_{\text {act }}$ by accessing data from the respective lowest energy ${ }^{1} P C^{*}$ (i.e., $S_{1}$ ) and ${ }^{3} P C^{*}$ (i.e., $T_{1}$ ) states. These four PCs have previously been the 
subject of detailed photophysical investigations, ${ }^{42,43}$ and findings in the polar solvent $N, N$ dimethylacetamide (DMAc) are summarized here.

Compounds 1 and 2 possess phenyl groups for $N$-aryl substituents which participate minimally in the photophysics. The $S_{1}$ of $\mathbf{1}$ is almost completely non-polar and is characterized by a delocalization of charge across the phenoxazine core and both core phenyl substituents (socalled S Deloc $)$. This state has a 3.24 ns lifetime marked by efficient fluorescent decay $\left(\Phi_{e m}=k_{r} /\left(k_{r}\right.\right.$ $\left.\left.+k_{n r}+k_{I S C}\right)=0.68\right)$ and a moderate yield of intersystem crossing $\left(\Phi_{I S C}=0.30\right)$ to a $\mathrm{T}_{1}$ state characterized by charge transfer from the phenoxazine core to a single core phenyl substituent

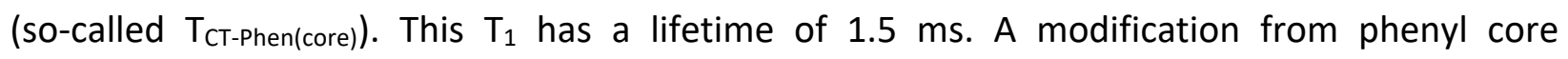
substituents to biphenyl substituents in $\mathbf{2}$ results in a slight redshift of its maximal wavelength of absorption and an increase in molar absorptivity (see Figure S\#1). That perturbation also imbues 2 with an $S_{1}$ state with partial CT character involving the core and a single biphenyl substituent;

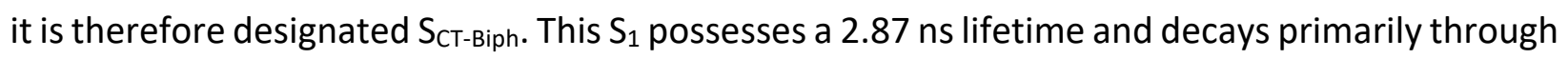
radiative means $\left(\Phi_{e m}=0.80\right)$. With modest yield $\left(\Phi_{I S C}=0.11\right)$ it forms a $T_{1}$ characterized by charge transfer from the phenoxazine to a single biphenyl substituent, denoted $\mathrm{T}_{\mathrm{CT}-\mathrm{Biph}}$, with a lifetime of $1.2 \mathrm{~ms}$.

In compounds $\mathbf{3}$ and $\mathbf{4}$, the $N$-aryl substituent is an $N$-naphthyl group. While this has little impact on the absorption spectrum or the nature of the lowest energy triplet excited states (relative to core-phenyl and core-biphenyl analogues, respectively), it markedly affects other photophysical behaviors. Formed following absorption into the Franck-Condon state, these compounds possess highly polar $S_{1}$ states characterized by a charge transfer from the phenoxazine to the orthogonal $N$-naphthyl substituent (so-called $\mathrm{S}_{\mathrm{CT}-\mathrm{Naph}}$ ). This electronic character slows radiative decay $\left(\Phi_{e m, 3}=0.019\right.$ and $\left.\Phi_{e m, 4}=0.023\right)$ while simultaneously speeding intersystem crossing, permitting near-unity $\Phi_{I S C}$ in both species $\left(\Phi_{I S C, 3}=0.95\right.$ and $\left.\Phi_{I S C, 4}=0.91\right)$. The $S_{1}$ lifetimes of 3 and 4 are $6.32 \mathrm{~ns}$ and $5.2 \mathrm{~ns}$, respectively and their $\mathrm{T}_{1}$ lifetimes are $1.5 \mathrm{~ms}$ and $0.48 \mathrm{~ms}$.

Measurement of Singlet $\boldsymbol{k}_{\text {act }}$ Values. Since the $S_{1}$ states in the four species are fluorescent, the shortening or quenching of $S_{1}$ lifetimes can be monitored using time-correlated single-photon 
counting (TCSPC). Quenching experiments were conducted in DMAc using the alkyl bromide initiator diethyl 2-bromo-2-methylmalonate (DBMM) as a quencher. DMAc and DBMM are commonly used for conducting O-ATRP.

In the absence of a reactive quencher the singlet lifetime, $\tau_{0, S_{1}}$, is defined by pathways ascribed to radiative and nonradiative decay, as well as intersystem crossing (Eq. 1a). In the presence of DBMM a bimolecular decay pathway is introduced, and the lifetime is shortened (Eq. 1b):

$$
\begin{array}{cc}
\tau_{0, S_{1}}=k_{0, S_{1}}^{-1}=\left(k_{r}+k_{n r}+k_{I S C}\right)^{-1} & \text { Eq. } 1 a \\
\tau_{S_{1}}=\left(k_{a c t}[D B M M]+k_{r}+k_{n r}+k_{I S C}\right)^{-1} & \text { Eq. } 1 b
\end{array}
$$

Inverting and rearranging Eq. $1 \mathrm{~b}$ yields a form of the Stern-Volmer equation ${ }^{48}$ :

$$
\frac{1}{\tau}-\frac{1}{\tau_{0}}=k_{a c t}[D B M M]
$$

A plot of $\frac{1}{\tau}-\frac{1}{\tau_{0}}$ data against [DBMM] should exhibit linear growth with a slope equal to the bimolecular rate coefficient, $k_{a c t}$, and a zero $y$-intercept. Conducting this analysis results in linear plots with slopes (i.e. $k_{\text {act }}$ values) ranging between $2.0 \times 10^{9} \mathrm{M}^{-1} \mathrm{~s}^{-1}$ and $4.80 \times 10^{9} \mathrm{M}^{-1} \mathrm{~s}^{-1}$. Diffusion places an upper limit on the rate of reaction. In DMAc at $20^{\circ} \mathrm{C}$ the diffusion-limited rate coefficient is estimated to be approximately $6.1 \times 10^{9} \mathrm{M}^{-1} \mathrm{~s}^{-1}$ using a simplified form of the Smoluchowksi equation. ${ }^{49,50}$ The measured singlet $k_{\text {act }}$ values determined from Fig. 2 approach this limit, but are still sensitive to the excited-state reactant properties. 


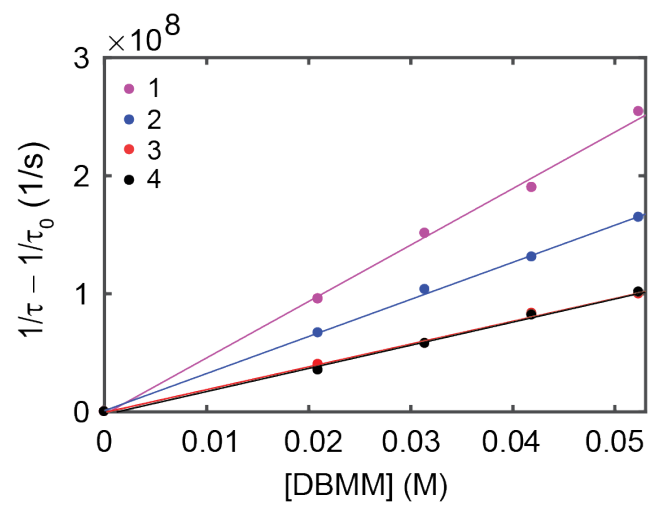

\begin{abstract}
Figure 2: Representative Stern-Volmer plots for $\mathrm{S}_{1}$ quenching experiments conducted on PCs 1-4, and their associated linear fits (solid lines). Each data set comprises of 5 concentration points, including one at [DBMM] = 0 (unquenched). The values and errors presented in Table 1 are a result of triplicate measurements.
\end{abstract}

Quenching of a singlet excited state is commonly attributed to either energy or electron transfer, but only the latter is expected in our system. Förster resonance energy transfer (FRET) requires spectral overlap between the emission (Figure S1) of the excited-state energy donor and the absorption spectrum of the acceptor. Since there is no absorption signal attributable to DBMM within the UV solvent window of DMAc $(268 \mathrm{~nm})$, and the PC emission occurs significantly to the red of $375 \mathrm{~nm}$, FRET is unlikely. Dexter energy transfer would require a dark singlet state for DBMM in resonance with the emitting PC $S_{1}$. To our knowledge, there is no reason to suspect this state is present for this alkyl-halide initiator. Therefore, electron transfer is the quenching pathway. Also, similar systems have been shown to react with alkyl-bromide O-ATRP initiators through dissociative electron transfer, supported by spectroscopic and EPR studies. ${ }^{39,40}$

The $S_{1}$ energy levels in Table 1 combined with redox data can be used to estimate driving forces for excited-state electron transfer. For the free energy available for reductive electron transfer by the $S_{1}$ excited state ${ }^{1} \mathrm{PC}^{*}$, the following equation is used:

$$
e E^{0}\left(P C^{\bullet+} /{ }^{1} P C^{*}\right)=e E^{0}\left(P C^{\bullet+} /{ }^{1} P C\right)-E_{00}
$$

Here $e$ is the fundamental charge, $E^{0}\left(P C^{\bullet+} /{ }^{1} P C\right)$ is the potential at which the $\mathrm{PC}$ radical cation is reduced to the ground state photocatalyst ${ }^{1} \mathrm{PC}$, and $E_{00}$ is the free energy stored in ${ }^{1} \mathrm{PC} *$ (measured through a vibronic fitting analysis of the fluorescence spectrum of a given PC-see SI 
for details). Once this is known, the driving force for electron transfer to a substrate, $\Delta G_{E T}^{0}$, can be calculated using Eq. 4:

$$
\Delta G_{E T}^{0}=e E^{0}\left(P C^{\bullet+} / P C^{*}\right)-e E^{0}\left(R X / R^{\bullet} X^{-}\right)-\frac{e^{2}}{4 \pi \varepsilon_{0} \varepsilon r}
$$

The second term in this equation involves the reduction potential of the alkyl halide ATRP initiator DBMM leading to the dissociated species. The final term is a Coulombic work term associated with the geminate ion pair ( $\varepsilon_{0}$ is the permittivity of free space, $\varepsilon$ is the relative permittivity of the dielectric medium [the solvent], and $r$ is the interionic distance following electron transfer). In the formation of encounter complexes in solution, the distance between reactants that engage in ET often falls between 6-10 $\AA$, including a solvation shell. ${ }^{49-51}$ For $r$ values within that range, the Coulombic term renders the ET process more exothermic by 37 to $62 \mathrm{meV}$ (see SI). While small in magnitude when compared to the rest of the equation, it is important for properly seating driving-force values within the greater ET literature. With this consideration in mind, a value of $-0.047 \mathrm{eV}$ was chosen, which corresponds to $r=8 \AA$, an intermediate value of the range quoted above.

All $\Delta G_{E T}^{0}$ values are listed in Table 1 where it is seen that PC 1 has the largest driving force $(-1.87 \mathrm{eV})$. Simultaneously, PC 1 exhibits the most rapid electron transfer of the four PCs with $k_{a c t}=4.80 \pm 0.09 \times 10^{9} \mathrm{M}^{-1} \mathrm{~s}^{-1}$, nearly at the diffusion-controlled limit. A decrease in the driving force

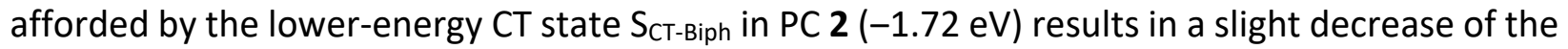
rate coefficient to $3.3 \pm 0.3 \times 10^{9} \mathrm{M}^{-1} \mathrm{~s}^{-1}$, following qualitative expectations of Marcus theory in the normal regime. PCs 4 and 3 with SCT-Naph states with even lesser driving force for ET (-1.62 eV and $-1.59 \mathrm{eV}$ ) follow this trend and participate in activation at a slower rate, $k_{\text {act }}=2.0 \pm 0.1 \times 10^{9} \mathrm{M}^{-1} \mathrm{~s}^{-1}$ for both. While all four PCs can react from their $S_{1}$ states, the overall efficacy with which they do so is still in question and will be influenced by the sub $10 \mathrm{~ns} \mathrm{~S}_{1}$ lifetimes. We will discuss results relevant to reactivity from the triplet manifold, then discuss the quantum efficiencies associated with both singlet and triplet spin manifolds.

Measurement of Triplet $\boldsymbol{k}_{\text {act }}$ Values. The $\mathrm{T}_{1}$ states of PCs 1-4 have sufficient driving force for ET (Table 1 and Figure 1) as well as lifetimes on the order of milliseconds (vide supra) that are 
long enough for many potentially productive collisions in solution. However, any one of those collisions should be less productive than a corresponding $S_{1}$ state collision due to the lower driving force for ET possessed by the triplet. This reasoning motivates an investigation of reactivity from the $T_{1}$ in PCs 1-4, which form in 11-95\% efficiency (Table 1 ) in the absence of quencher. Prior DFT studies show that all four $\mathrm{T}_{1}$ states are CT in character, with the upper singlyoccupied molecular orbital (SOMO) residing on a single phenyl (PCs 1,3) or biphenyl core substituent (PCs 2,4), and the lower SOMO on the phenoxazine core, termed TCT-Phen and TCT-Biph respectively. ${ }^{22} \mathrm{~N}$-aryl substitutional differences have a negligible effect on computed $\mathrm{T}_{1}$ energies (computation of the energy is required because phosphorescence has not been observed) and little effect on the resultant $\Delta G_{E T}^{0}$. These values as listed in Table 1 are determined using Eq. 4 and substituting in a DFT-calculated excited-state reduction potential for $E^{0}\left(P C^{\bullet+} /{ }^{3} P C^{*}\right)$. In agreement with the predicted orbital characteristics, those PCs sharing common core substituents ( $\mathbf{1}$ with $\mathbf{3}$ as well as $\mathbf{2}$ with $\mathbf{4}$ ) have nearly identical excited-state absorption (ESA) features as observed in TA experiments: core-biphenyl PCs $\mathbf{2}$ and $\mathbf{4}$ show a maximum in their ESA at $700 \mathrm{~nm}$, and core-phenyl PCs 1 and 3 show an ESA maximum at $650 \mathrm{~nm}$.

We rule out the possibility of triplet energy transfer (TET) from the phenoxazine $T_{1}$ state to DBMM being responsible for quenching by appealing to the triplet energies of both reactants. Efficient (diffusion-limited) TET occurs when the triplet energy of the donor exceeds that of the acceptor by at least $0.125 \mathrm{eV} .{ }^{41}$ DBMM has been sensitized in the past by $p$-anisaldehyde, which possesses a triplet energy of $3.125 \mathrm{eV} .{ }^{52}$ In that system, energy transfer to DBMM precedes homolytic cleavage of the $\mathrm{R}-\mathrm{Br}$ bond, and so the bond-dissociation energy (BDE) can be taken as a lower bound for the triplet energy. As an estimate, the R-Br BDE for an analog of DBMM, diethyl 2-bromomalonate has been measured at $2.75 \mathrm{eV}{ }^{53}$ TET from these phenoxazines (with calculated triplet energies between $2.11 \mathrm{eV}$ and $2.35 \mathrm{eV}$ ) to DBMM is then expected to be endergonic by $\sim 0.5 \mathrm{eV}$ at the least using the quoted BDE as an estimate, and so any observed quenching of the triplet states should be attributed to electron transfer.

Stern-Volmer quenching studies were conducted by monitoring lifetime shortening at the ESA of a given $T_{1}$ state as a function of [DBMM]. Notably, the DBMM concentration range is approximately three orders of magnitude lower in these experiments compared with the singlet 
studies discussed above that utilized values closer to common O-ATRP conditions. The four $k_{\text {act }}$ values (Table 1) partition into two groups of similar values. PCs $\mathbf{1}$ and $\mathbf{3}$, with core-phenyl substituents, highly similar electronic properties in the $T_{1}$, and a common value of $\Delta G_{E T}^{0} \approx-1.34$ $\mathrm{eV}$, display $k_{\text {act }}$ values that are within the error of our measurements at $1.9 \pm 0.1 \times 10^{8} \mathrm{M}^{-1} \mathrm{~s}^{-1}$ and $2.0 \pm 0.1 \times 10^{8} \mathrm{M}^{-1} \mathrm{~s}^{-1}$, respectively. Similarly, PCs 2 and 4 with core-biphenyl substituents with $\Delta G_{E T}^{0}$ $\approx-1.17 \mathrm{eV}$, yield $k_{\text {act }}$ values of $1.20 \pm 0.09 \times 10^{8} \mathrm{M}^{-1} \mathrm{~s}^{-1}$ and $1.10 \pm 0.09 \times 10^{8} \mathrm{M}^{-1} \mathrm{~s}^{-1}$, respectively. Like the singlet data, the triplet data qualitatively follow the expectations of electron transfer in the Marcus-normal regime, with greater driving force leading to more rapid electron transfer. In addition to triplet lifetime shortening upon addition of DBMM, the TA measurements contain signal associated with the phenoxazine radical cation, which presents as a persistent excitedstate-absorption signal that does not decay on the timescale of the experiment. Spectroscopic signatures of the radical cation are known from prior spectroelectrochemistry experiments and overlap at all wavelengths that were accessible for measurement of the triplet signals. These observations corroborate the expectation that quenching is due to electron transfer and not to Dexter energy transfer from the $\mathrm{PC}_{1}$.

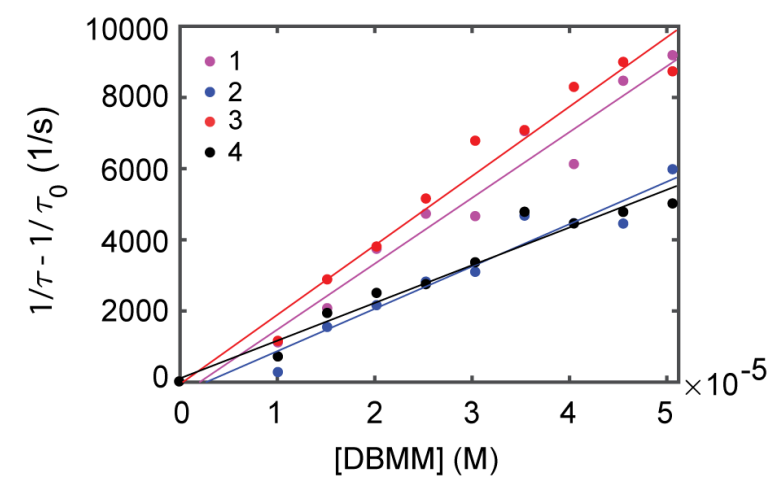

Figure 3: Stern-Volmer plots for $\mathrm{T}_{1}$ quenching experiments conducted with PCs 1-4, and their associated linear fits. Each data set comprises 10 concentration points, including one at $[\mathrm{DBMM}]=0$ (unquenched).

Driving-Force Relationship. Our data (Table 1) span a driving-force range of nearly $1 \mathrm{~V}$, over which the corresponding $k_{\text {act }}$ values show a noticeable dependence. We sought to unify these observations in the application of the Marcus Theory of Electron Transfer. The effects of diffusion are explicitly included when considering a bimolecular system, since it is a process with 
a competitive rate coefficient and can have drastic effects on the predicted behavior of the system. The following kinetic scheme is employed:

$$
\mathrm{PC}^{*}+\mathrm{R}-\mathrm{Br} \underset{\mathrm{k}_{\text {-diff }} \stackrel{\mathrm{k}_{\text {diff }}}{\rightleftarrows}}{\longrightarrow} \mathrm{PC}^{*} \cdots \mathrm{R}-\mathrm{Br} \stackrel{\mathrm{k}_{\mathrm{ET}}}{\longrightarrow} \mathrm{PC}^{*+}+\mathrm{R}^{*}+\mathrm{Br}^{-}
$$

Scheme 2: Kinetic scheme necessary for describing kact.

The excited-state PC (irrespective of spin multiplicity) and initiator first diffuse together $\left(k_{\text {diff }}\right)$ and form an encounter complex. After the encounter complex is generated, it can either break apart $\left(k_{\text {-diff }}\right)$ or engage in dissociative electron transfer $\left(k_{E T}\right)$. The electron-transfer portion of this scheme is simplified to draw focus to the forward electron transfer process. In the treatment of experimental yields there are additional processes that need to be considered (vide infra). Within this scheme, the diffusion-limited rate coefficient $\left(k_{\text {diff }}\right)$ can be estimated using a simplified form ${ }^{50}$ of the Smoluchowski equation:

$$
k_{\text {diff }}=\frac{8 R T}{3 \eta}
$$

This equation allows for the estimation of the diffusion-limited rate coefficient in roomtemperature DMAc using its viscosity $\eta_{,}^{54}$ the ideal gas constant $R$, and the temperature $T$. Using these values produces a value of $k_{\text {diff }}=6.1 \times 10^{9} \mathrm{M}^{-1} \mathrm{~s}^{-1}$. The rate coefficient associated with reactants departing, $k_{\text {-diff, }}$ is related to $k_{\text {diff }}$ through an equilibrium constant ${ }^{49-51}: k_{d}=k_{\text {difff }} / k_{\text {-diff. }}$. For two nonpolar, uncharged reactants such as any of these PC/DBMM pairs that engage in electron transfer with typical encounter distances between $5 \AA$ and $8.5 \AA, K_{d}$ is expected to take on values between $0.38 \mathrm{M}^{-1}$ and $1.1 \mathrm{M}^{-1}$ owing to a reliance on the center-to-center distance of the two reactants in the encounter complex-details of this calculation are presented in the SI. Inputting the same center-to-center distance used previously for estimating the Coulombic work term in $\Delta G_{E T}^{0}$, we estimate $K_{d}=0.55 \mathrm{M}^{-1}$.

We utilize the classical Marcus theory expression for the electron-transfer rate coefficient, $k_{E T}$, based on its successful application across a variety of organic dissociative electron 
transfer systems, including ones that feature aromatic radical anions, transition metal complexes, and solvated electrons as reductants. ${ }^{49,55}$

$$
k_{E T}=\frac{k_{B} T}{h} \exp \left[-\frac{\Delta G_{E T}^{\ddagger}}{k_{B} T}\right]
$$

Eq. 6 is the Eyring equation with a unity transmission coefficient ( $Z=1$, not shown), and an activation energy, $\Delta G_{E T}^{\ddagger}$, given by:

$$
\Delta G_{E T}^{\ddagger}=\frac{\left(\Delta G_{E T}^{0}+\lambda_{o}+B D E\right)^{2}}{4\left(\lambda_{o}+B D E\right)}
$$

Here $\lambda_{0}$ is the "outer-sphere" reorganizational energy associated with fluctuations of the solvent orientation and polarizability, and BDE refers to the alkyl-halide bond-dissociation energy. In Eq. 7 , the definition of the total reorganization energy takes on a form attributed to Savéant in descriptions of dissociative-electron transfer theory. ${ }^{56-58}$ Namely, in standard Marcus theory the total reorganization energy is defined as a sum of outer and inner-sphere contributions $\left(\lambda=\lambda_{o}+\right.$ $\left.\lambda_{i}\right)$, whereas in Marcus-Savéant theory, the inner sphere contributions are subsumed in the energetic cost associated with bond cleavage (BDE).

The collective behavior of $k_{\text {diff }}$ and $k_{E T}$ can be described by translating Scheme 2 into a set of kinetic equations and applying the steady-state approximation to the encounter complex. In doing this, a composite rate coefficient ${ }^{59}$ emerges that connects the quenching rate coefficients determined from Stern-Volmer studies $\left(k_{\text {act }}\right)$ to the driving force for electron transfer, which is embedded in $k_{E T}$ (via Eq. 6):

$$
k_{a c t}=\frac{k_{\text {diff }}}{1+\left(\frac{k_{\text {diff }}}{K_{d}}\right)\left(\frac{h}{k_{B} T}\right) \exp \left[\frac{\left(\Delta G_{E T}^{0}+\lambda\right)^{2}}{4 \lambda k_{B} T}\right]}
$$

A derivation of this formula is provided in the SI.

Fig. 4 shows a plot of $k_{a c t}$ versus $\Delta G_{E T}^{0}$ for the eight data points from compounds 1-4 (again, both singlet and triplet reactivity). Modelling using Eq. 8 (solid line) is possible with the reorganization energy as a single fitting parameter. Using $k_{\text {diff }}=6.1 \times 10^{9} \mathrm{M}^{-1} \mathrm{~s}^{-1}$ and $K_{d}=0.55 \mathrm{M}^{-1}$ as justified previously, we find $\lambda=3.10 \pm 0.02 \mathrm{eV}$. Also shown in Fig. 4 are data from several 
literature reports of $k_{\text {act }}$ including two points with larger $\Delta G_{E T}^{0}$ than is accessible for $\mathbf{1 - 4}$. The data points marked by hollow circles correspond to work by Jockusch and Yagci who measured kact values for quenching of the singlet and triplet state of a phenothiazine-based PC by the alkylbromide ATRP initiators methyl $\alpha$-bromoisobutyrate (MBI) and ethyl $\alpha$-bromophenylacetate (EBPA) in DMAc. ${ }^{40}$ The data point marked by a hollow square comes from Matyjaszewski and coworkers, who measured the quenching of a different phenothiazine PC by EBPA. ${ }^{35}$ In the case of the full data set ( $k_{\text {act }}$ for $1-4$ plus the literature values), fitting indicates $\lambda=3.09 \pm 0.04 \mathrm{eV}$. It is emphasized that this fit is visually indistinguishable from the fit of the 1-4 data set and is not separately included. Overall, the quality of fit to a data set inclusive of rate constants from a significant variety of PCs and quenchers is compelling. Also compelling is the close agreement of $\lambda$ values when modeling with only data from 1-4 versus data from a larger range of $\Delta G_{E T}^{0}$.

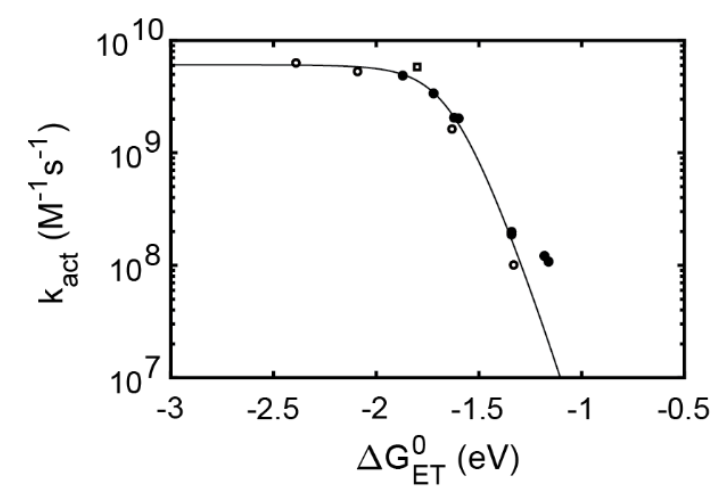

\footnotetext{
Figure 4: The driving force dependence of $k_{a c t}$ as observed for our systems (black) as well as values reported by Yagci (hollow circles) and Matyjaszewski (hollow square). The solid line is a fit of Eq. 8 to only the data from this work (full circles), with $\mathrm{K}_{\mathrm{d}}=0.55 \mathrm{M}^{-1}, \lambda=3.10 \mathrm{eV}$, and $k_{\text {diff }}=$ $6.1 \times 10^{9} \mathrm{M}^{-1} \mathrm{~s}^{-1}$.
}

The value of $\lambda=3.1 \mathrm{eV}$ that we find is of the right magnitude for a dissociative electron transfer in this type of system given that a large portion comes from the alkyl-bromide bonddissociation energy of DBMM $\left(\lambda=\lambda_{o}+B D E\right)$. The other portion comes from the outer-sphere reorganization energy, for which we assume $0.65 \mathrm{eV}$. This value is the average of several values estimated by Savéant for dissociative electron transfers involving several alkyl bromides in a similar solvent, $N, N$-dimethylformamide (DMF). ${ }^{56} U \operatorname{sing} \lambda_{0}=0.65 \mathrm{eV}$ results in a bond-dissociation energy of $2.45 \mathrm{eV}$ for the $\mathrm{R}-\mathrm{Br}$ bond in $\mathrm{DBMM}$. This value is similar to a literature value for the alkyl-bromide BDE of DBM, which is $2.75 \mathrm{eV} \cdot{ }^{53}$ Additionally, the previously mentioned alkyl- 
bromide initiators EBPA and MBI have been the subject of a DFT study in which their bonddissociation energies were calculated at $2.55 \mathrm{eV}$ and $2.34 \mathrm{eV}$ respectively. ${ }^{38}$ We suspect that a similar study conducted on DBMM would result in an intermediate bond-dissociation energy with respect to the two. $\mathrm{MBI}$ has a single adjacent ester group to delocalize the radical across, while the radical formed on EBPA has an adjacent ester and benzene ring. With two adjacent esters, DBMM should act as an intermediate in terms of radical stabilization.

As a secondary point, it is reiterated that we set out to consider whether there are demonstrable effects of electronic character (for example the degree of charge transfer) and spin on the dissociative electron transfer process. Notably the successful application of classical Marcus theory (Eq. 8) argues against this possibility. To further explore this point, the full data set was modeled using a modified version of Eq. 8 that contains the semiclassical Marcus theory expression for $k_{E T}(E q . S 12 a, b)$ that includes the diabatic electronic coupling $V$ between reactant and product states. In our modeling, the same value of $K_{d}$ was used, and $\lambda$ was set to $3.1 \mathrm{eV}$ as determined from the previous classical fitting process. This approach left the electronic coupling as the only fitting coefficient, resulting in a value of $V=25.3 \pm 1.5 \mathrm{meV}$. A value of $V$ that is the same as room temperature $k_{B} T\left(25.3 \mathrm{meV}\right.$ at $\left.20^{\circ} \mathrm{C}\right)$ points to a case where adiabatic (classical) theory is appropriate but where semiclassical behavior is not far off. To the former point, Savéant has also observed electronic coupling of similar magnitude with the application of a classical treatment in a dissociative electron transfer study involving aromatic singlet excited-state reductants and carbon tetrachloride as a dissociative substrate. ${ }^{60}$ It is likely that the rate coefficient map of Fig. 4 can be applied to many systems. To the latter point, semiclassical considerations may be warranted in cases of significant reactant sterics and qualitatively different electronic character of the reactant electron donor.

Three regimes of note are present in the Fig. 4 data and fits. The first regime is at lower driving forces, for example between $-1 \mathrm{eV}$ and $-1.5 \mathrm{eV}$, where the triplet $k_{a c t}$ values from our study lie. In this regime, electron transfer is rate limiting and the rate constant for diffusion ( $\left.k_{\text {diff }}\right)$ has limited impact on $k_{a c t}$, allowing for its estimation to be largely made by appealing to $k_{E T}$ alone: in the limit of $K_{d} k_{E T}$ " $k_{\text {diff, }}$, then $1+k_{\text {difff }} / K_{d} k_{E T} \approx k_{\text {difff }} / K_{d} k_{E T}$ and subsequently $k_{a c t} \approx K_{d} k_{E T}$ (via Eq. 8). The second regime is in the other driving-force extreme, where $\Delta G_{E T}^{0}$ is more negative than -2 
eV. Here $K_{d} k_{E T} \gg k_{\text {diff }}$ and $k_{\text {act }}$ approaches diffusion-limited behavior: $k_{a c t}=k_{\text {diff. }}$ This phenomenon is known as diffusional leveling, and it is notable that the values included from Jockusch and Yagci pertaining to their phenothiazine's strongly reducing singlet state fall into that second regime. Diffusion control is expected to continue until much greater driving force values, at which point a decrease in rate coefficient associated with entrance into the Marcus inverted regime may be observed. ${ }^{61}$ Alternatively, those strongly exoergic electron transfers can lend themselves to the formation of a metastable alkyl-halide radical anion, in which case there are different thermodynamic and kinetic considerations in place, though such discussion is outside the scope of this work. ${ }^{57,58}$ The third regime is between the two previous ones, where $k_{\text {act }}$ is sensitive to both ET as well as diffusion. Our study's singlet data sits within this region. Overall, this modeling provides a persuasive retrospective estimation of literature data, including ours, with all values falling within at most a factor of two of the value predicted by the Marcus curve (solid line) in Fig. 4. Providing a driving-force range map is useful for addressing how a PC excited state-be it singlet or triplet-interacts with the activation process in O-ATRP. Importantly, this map appears useful irrespective of the electronic character (CT, LE) or spin character (singlet, triplet) of the excited state electron donor.

Competitive Reactivity: Singlets vs. Triplets. In the field of O-ATRP a question is commonly raised as to the nature of the catalytically relevant excited state. In our systems, where photophysical control is engendered by synthetic control of PC structure, measured $k_{a c t}$ values can address this concept. PCs $\mathbf{2}$ and $\mathbf{4}$ have been chosen for consideration for two reasons. First, they have disparate $\Phi_{I S C}$ values of 0.11 vs. 0.91, respectively. Second, they have similar electronic character, as discussed in the Photophysical Background section, characterized as CT in nature involving charge shifting from the phenoxazine core to one of the biphenyl substituents. Thus, a comparison of these species helps to isolate the impact of spin (this comparison is discussed further in the final section of this paper). Irrespective of spin or electronic character of the reactant state, although relying on its lifetime $\tau_{0}=\left(\mathrm{k}_{0}\right)^{-1}$, the quantum efficiency of activation from an excited state as a function of [DBMM] is written below in Eq. 9A: 


$$
\begin{gathered}
\phi_{a c t}=\frac{k_{a c t}[D B M M]}{k_{a c t}[D B M M]+k_{0}} \\
\Phi_{a c t, S 1}=\phi_{a c t, S 1} \\
\Phi_{a c t, T 1}=\Phi_{I S C} \phi_{a c t, T 1} \\
\Phi_{I S C}=\frac{k_{I S C}}{k_{0, S 1}+k_{a c t}[D B M M]}
\end{gathered}
$$

For the $S_{1}$, which is assumed to be produced in unit quantum yield following light absorption, the overall quantum yield for activation $\left(\Phi_{a c t, S_{1}}\right)$ is equivalent to this quantum efficiency of activation (Eq. 9B). For the $T_{1}$, the overall quantum yield for activation $\left(\Phi_{\text {act, }, T_{1}}\right)$ must be modified with the yield of intersystem crossing to account for competing non-ET loss processes following light absorption (Eq. 9C). It is important to recognize that $\Phi_{\text {ISC }}$ can be thought of as a constant (the value given in Table 1) so long as [DBMM] is low enough that $S_{1}$ states will not encounter a quencher molecule. If [DBMM] is sufficiently large that $S_{1}$ quenching is expected, $\Phi_{\text {Isc }}$ will be reduced due to competitive quenching (Eq. 9D). The quantities $\Phi_{a c t, S 1}$ and $\Phi_{a c t, T 1}$ have been calculated as a function of [DBMM] and are shown for PC $\mathbf{2}$ and $\mathbf{4}$ in Fig. $5 \mathrm{~A}$ and B, respectively. The overlaid green line in the plots shows a commonly employed DBMM concentration for OATRP: $[\mathrm{DBMM}]_{\mathrm{synth}}=45 \mathrm{mM}$.

As seen with the dashed lines, for both compounds the $T_{1}$ state is the only participant in activation at values of [DBMM] below $1 \mathrm{mM}$. This role maximizes with a yield of activation almost equivalent to the respective unquenched $\Phi_{\text {Isc }}$ before decreasing due to the onset of singlet-state reactivity which competes with the intersystem crossing process (see Eq. 9D). For 2, which was chosen because of its low $\Phi_{I S C}$, a turnover in reactive-state identity occurs at about $10 \mathrm{mM}$, after which $S_{1}$ reactivity is the more important consideration. For 4 , given its near-unity $\Phi_{I S C}$, O-ATRP activation is expected to be efficient even at 10-100 micromolar concentrations of initiator. This efficiency continues to increase as initiator is added and by [DBMM] synth activation is nearly quantitative. At that concentration, the $T_{1}$ is expected to be responsible for twice as many activation reactions as the $S_{1}$, making it the major catalytic state for PC 4 under synthetic conditions. It is important to note that even at $[D B M M]_{\text {synth }}$ this is not the case with PC $\mathbf{2}$, which is primarily reactive from its $S_{1}$ due to its poor $\Phi_{I S C}$. Because of this, much greater [DBMM] would 
be necessary for PC 2 to achieve the same total activation efficiency of PC 4. While high $\Phi_{I S C}$ is a promoter of $\mathrm{T}_{1}$ state reactivity it is important to recognize that $\mathrm{S}_{1}$ reactivity should not be ignored. While there are certainly differences in the reactive behavior of the two photocatalysts, there is not massive disparity in the proportion of state reactivity at $[D B M M]_{\text {synth: }} T_{1}$ versus $S_{1}$ reactivity occurs with a ratio of $1: 4$ and 2:1 for 2 and 4 respectively. An analogous result comes from conducting this analysis on PCS $\mathbf{1}$ and 3, which have similar relative $\Phi_{I S C}$ values to $\mathbf{2}$ and $\mathbf{4}$.
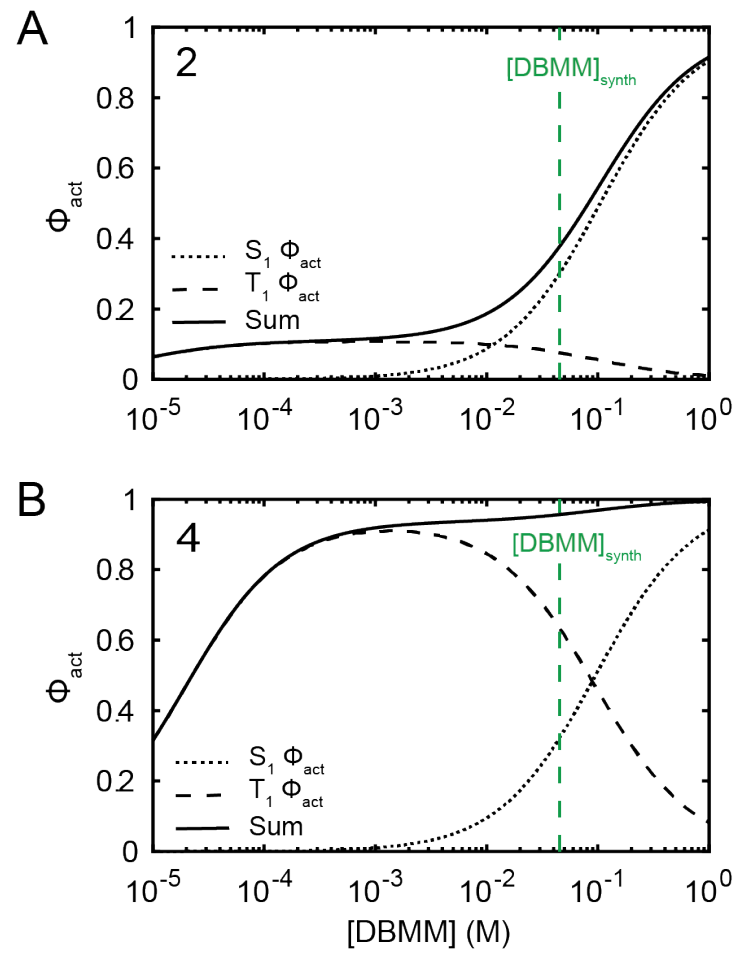

\begin{abstract}
Figure 5: Semilogarithmic plots of the calculated (Eqs. 9A-D) quantum yields of activation from the $S_{1}$ state, $T_{1}$ state, and the sum of the two for PCs 2 (A) and 4 (B) as a function of [DBMM]. PC 2's low predicted yield at lower values of DBMM reflects its poor ability to form its long-lived $T_{1}$, while the near-unity triplet yield of $\mathbf{4}$ engenders it with uniform reactivity over a large concentration range.
\end{abstract}

\title{
Photochemical Quantum Yield of Dissociative Electron Transfer
}

While Fig. 5 is compelling for understanding origins of reactivity under various reaction conditions, it is based on observations of electron-transfer quenching and ultimately does not speak to the possibility of spin-state dependencies in photochemical product yield arising because of competition with non-productive pathways such as back electron transfer. In this 
section we address this question and describe experiments to measure the quantum yield of productive dissociative electron transfer to form the fragmented products $\left(\mathrm{PC}^{\bullet+}, \mathrm{R}^{\bullet}\right.$, and $\left.\mathrm{Br}^{-}\right)$.

Photochemical quantum yields are measured from the vantage point of ground state species as a ratio of the moles of photoproduct generated over a period of illumination with the moles of photons absorbed over that same period:

$$
\Phi_{r \times n}=\frac{\text { moles of photoproduct formed }}{\text { moles of photons absorbed }}
$$

The kinetics of a photoreaction can be complicated, however they are simplified and forced into a zeroth order regime (see the SI for an extensive discussion, including kinetic simulations of the system) when the concentration of the absorbing species is large enough that more than $99 \%$ of the photons incident on the reaction mixture are absorbed. ${ }^{62-64}$ In general, the rate of photon absorption at a specific wavelength, $W(\lambda)$, can be written as:

$$
W(\lambda)=I_{0}(\lambda)\left(1-10^{-A(\lambda)}\right)
$$

where $I_{0}(\lambda)$ is the intensity of excitation light with wavelength $\lambda$ incident on the reaction mixture, and $A(\lambda)$ is the photoreactant absorbance at that same wavelength. In the high-absorbance limit (typically taken to be $A>2$ ), Eq. 11 simplifies since the exponential term can be omitted, resulting in $W(\lambda)=I_{0}(\lambda)$. Hence, so long as the sample absorbance fits this criterion, the moles of photons absorbed over a certain time period can be calculated as $I_{0}(\lambda) t$, so long as the intensity $I_{0}(\lambda)$ is known. This intensity measurement can be conducted with a calibrated power meter or with a chemical actinometer. We opted for the former and detail that measurement in the SI.

The most straightforward means of observing photoproduct generation in these systems is via the absorption signal of the generated PC radical cation, which exhibits multiple bright absorption bands in the visible spectrum (Fig. 6). The radical cation concentration, [c], can in principle be calculated given an absorbance measurement at a wavelength devoid of signal from other species. Initial attempts were made using spectroelectrochemical conversion to determine spectra and corresponding molar attenuation coefficients for the radical cations in DMAc. 
However, these measurements proved intractable due to incomplete transformation from PC to $\mathrm{PC}^{\bullet+}$ within the spectroscopic volume. The radical cations show a propensity for loss by oxidation of DMAc in the dark and more rapidly upon illumination. To overcome this challenge, we identified acetonitrile as a more oxidatively stable solvent with a comparable dielectric constant to DMAc and turned to chemical methods for quantitative generation of radical cations $1 \mathrm{c}, \mathbf{2 c}$, and $\mathbf{4 c}$ (synthetic details in the $\mathrm{SI}$ ). With these bench-stable radical cations in hand, molar attenuation coefficient values were measured for all three compounds via a Beer's law study (see SI). The absorption spectra (Fig. 6) of $\mathbf{2 c}$ and 4c, which both contain biphenyl core substituents, are quite similar possessing a dominant, broad, near-IR peak centered at $808 \mathrm{~nm}\left(\varepsilon_{808,2 \mathrm{c}}=12600\right.$ $\left.\mathrm{cm}^{-1} \mathrm{M}^{-1}\right)$ and $830 \mathrm{~nm}\left(\varepsilon_{830,4 \mathrm{c}}=14300 \mathrm{~cm}^{-1} \mathrm{M}^{-1}\right)$ respectively, and a less intense bluer feature that peaks near $480 \mathrm{~nm}$. The spectrum for $\mathbf{1 c}$ shows certain similarities to $\mathbf{2 c}$ and $\mathbf{4 c}$, although features are blue shifted owing to the smaller aromatic core substituents. A broad intense band peaks at $728 \mathrm{~nm}\left(\varepsilon_{728,1 \mathrm{c}}=17700 \mathrm{~cm}^{-1} \mathrm{M}^{-1}\right)$ and there is a double-peaked band near $450 \mathrm{~nm}$. Additionally, a band at $360 \mathrm{~nm}$ is resolved for $\mathbf{1 c}$, which is in a similar position to that of the parent PC. Because the lowest energy absorption peaks of the radical cations do not overlap with absorption or emission signatures of the respective parent PCs (see Fig. S1), they permit observations that can be ascribed purely to product generation during a photoreaction.

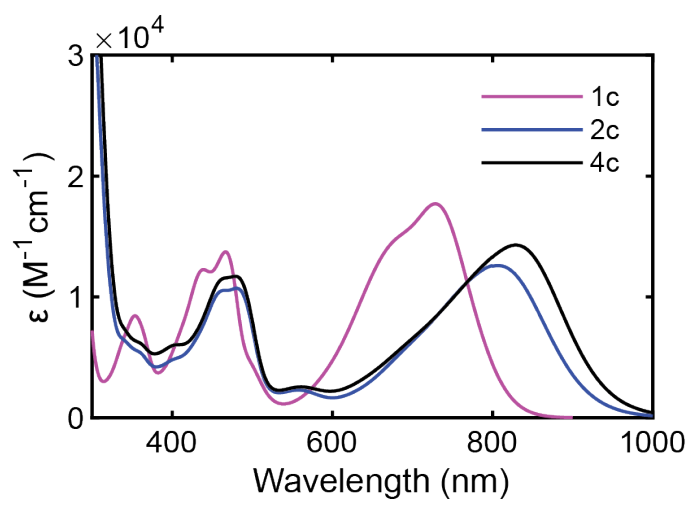

Figure 6: Absorption spectra of the radical cation hexafluorophosphate salts, collected in MeCN, scaled to their peak molar attenuation coefficients. Beer's law studies are presented in the SI.

As noted earlier and illustrated in Fig. 5, the choice of PC system and initiator concentration allows us to control reactant qualities such as the spin state engaged in 
photochemistry. We first target quantification of a reaction quantum yield involving singlet reactivity by considering PC 2 with a high DBMM concentration of $1 \mathrm{M}$. Illustrated in Fig. 7A is the growth of the absorption spectrum of $2 \mathrm{c}$ over an irradiation period of 60 seconds. The corresponding plot of [2c] vs. t (Fig. 7B) shows the linear growth expected from forcing this photoreaction into a zeroth order kinetic regime. The slope $(m)$ of the linear fit to this data gives the rate of $[\mathbf{2 c}]$ generation in units of $M / s$. As mentioned previously, at sufficiently high absorbances the rate of photon absorption (in units of $\mathrm{M} / \mathrm{s}$ ) is constant and so the total number of photons absorbed over a period $(t)$ is given by $I_{0} t$. Thus, the quantum yield of radical cation generation (i.e., Eq. 10: (mols of photoproduct)/(mols of photons absorbed)) can be determined as:

$$
\Phi_{r \times n}=\frac{m t}{I_{0} t}=\frac{m}{I_{0}}
$$

We find for 2 a value of $\Phi_{r \times n}=0.64 \pm 0.04$ (Table 2 )

Because the quantity $\Phi_{r x n}$ is referenced relative to ground state species that absorb light, and not relative to the specific states doing the dissociative electron transfer, it is important to take a step further and quantify the reaction yield in a way that accounts for competing photophysical and/or photochemical pathways. In a general way we can write in Eq. 13A that the quantum yield of reaction can be broken into a product of the quantum yield for activation $\left(\Phi_{\text {act }}\right)$ - where activation is thought of as the bimolecular quenching phenomenon-and the yield of productive dissociative electron transfer that generates an observed radical cation $\left(\Phi_{+}\right)$. In this way, it is seen that the reaction yield depends not only on the quenching events carried in $\Phi_{\text {act }}$ but also on how the excited state reactant enables production of the radical cation product.

$$
\begin{gathered}
\Phi_{r \times n}=\Phi_{a c t} \Phi_{+} \\
\Phi_{+, S 1}=\Phi_{r \times n, S 1} / \Phi_{a c t, S 1}=\Phi_{r \times n, S 1} / \phi_{a c t, S 1} \\
\Phi_{+, T 1}=\Phi_{r \times n, T 1} / \Phi_{a c t, T 1}=\Phi_{r \times n, T 1} / \Phi_{I S C} \phi_{a c t, T 1}
\end{gathered}
$$

Then, by appealing to Eqs. $9 \mathrm{~B}$ and $9 \mathrm{C}$ we can write reaction yield expressions that are specific to the states engaging in them. For 2 under high [DBMM] conditions, the specific state engaging in 
the photochemistry is the $S_{1}$ and we appeal to Eq. 13B. The quantum yield of $S_{1}$ formation is unity, however electron transfer is in meaningful competition with radiative decay such that at $1 \mathrm{M}$ DBMM, $\Phi_{a c t, S 1}=\phi_{a c t, S 1}=0.9$ (Table 2). Thus it is found that $\Phi_{+, S 1}=0.71 \pm 0.04$.
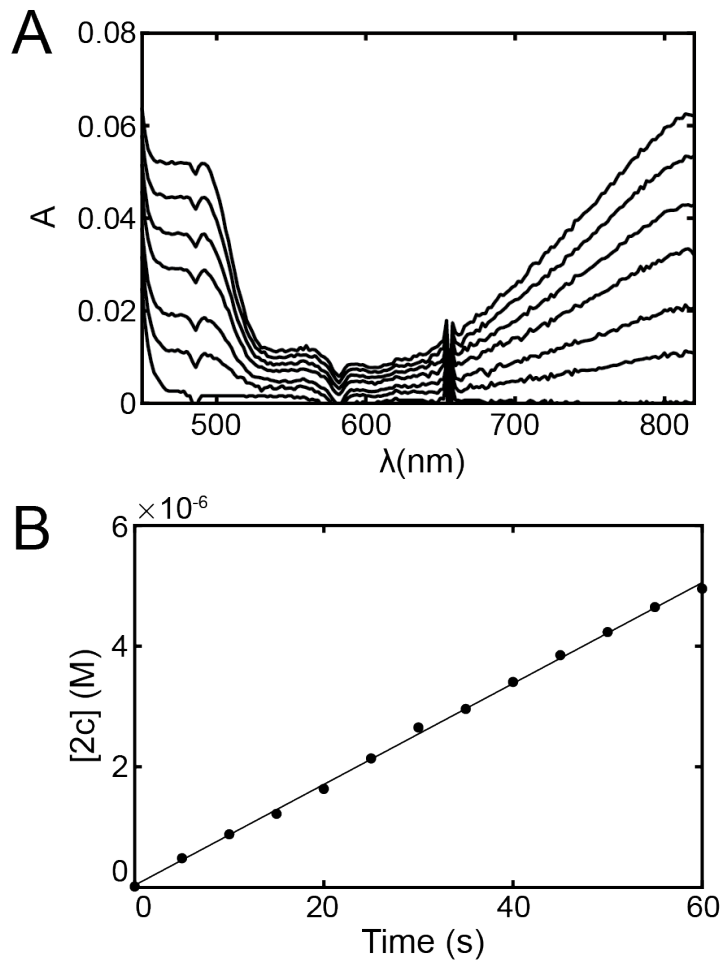

Figure 7: (A) Growth of the absorption signal of 2c over time in a photoreaction. (B) Kinetic trace at $816 \mathrm{~nm}$ converted to [2c] via Beer's law, with a linear fit demonstrating its zeroth order kinetic behavior.

Turning to a triplet excited state reactor in 4 at $1 \mathrm{mM} \mathrm{DBMM}$, we again find linear growth in 4c (Fig. S8C) and using Eq. 12 find a remarkably similar $\Phi_{r \times n, T 1}=0.65 \pm 0.02$ (Table 2). Given that $\Phi_{\text {Isc }}$ is not unity (it is 0.91 ; Table 1 ), but recognizing that the quantum efficiency of activation for this state once formed is unity ( $\phi_{a c t, T 1}$; Eq. 9A), we find $\Phi_{+, T 1}=0.71 \pm 0.07$ (Table 2), an identical finding to that of the PC 2's $S_{1}$. This finding is highly notable given that the electronic character of the $T_{1}$ in 4 is expected to be the same as the $S_{1}$ in $\mathbf{2}$ with CT manifesting from the phenoxazine core to one of the biphenyl substituents (vide supra). 
Thus, to the extent that we have controlled for and eliminated complications from electronic or other unforeseen factors, we can conclude there is no spin dependence on productive dissociative electron transfer. As the forward electron transfer event is manifest in the events of quenching and quantified with $\Phi_{a c t}$, this current finding in $\Phi_{+}$suggests no meaningful spin dependence in subsequent events that compete with photoproduct formation. Potentially competing events include (a) DMAc oxidation by $\mathrm{PC}^{\bullet+}$ which we know to be operative, albeit yet unquantified, from our spectroelectrochemical studies (vide supra) and (b) back electron transfer events wherein the photoproducts $\mathrm{PC}^{\bullet+}, \mathrm{R}^{\bullet}$ (the DBMM initiator following halide loss), and $\mathrm{Br}^{-}$recombine to generate singlet ground state species PC and DBMM. Because the one-electron reduction potentials ${ }^{22}\left(\mathrm{PC}^{\bullet+} / \mathrm{PC}\right)$ are nearly identical for $\mathbf{2}$ and $\mathbf{4}$, we can conclude that there is no evidence for spin-dependent loss via back electron transfer. It is noted that $\Phi_{r x n, S 1}$ was also measured for $\mathbf{1}$, as shown in Table 2 . That system is discussed in the conclusion.

Table 2: Values Related to the Measurement of Photochemical Quantum Yields

\begin{tabular}{|c|c|c|c|c|c|}
\hline PC & $\lambda_{\max }(\mathrm{nm})$ for $\mathrm{PC}^{\bullet+a}$ & $\varepsilon_{\lambda_{\max }}\left(\mathrm{M}^{-1} \mathrm{~cm}^{-1}\right)^{a}$ & $\Phi_{a c t}^{b}$ & $\Phi_{r x n^{c}}$ & $\Phi_{+}{ }^{d}$ \\
\hline 1 & 728 & $17700 \pm 600$ & 0.94 & $0.42 \pm 0.05$ & $0.45 \pm 0.05$ \\
\hline 2 & 808 & $12600 \pm 400$ & 0.90 & $0.64 \pm 0.04$ & $0.71 \pm 0.04$ \\
\hline 4 & 830 & $14300 \pm 900$ & 1.0 & $0.65 \pm 0.02$ & $0.71 \pm 0.07$ \\
\hline
\end{tabular}

${ }^{a}$ In MeCN. Error bars come from regression analysis of the Beer's law plots. ${ }^{b}$ Calculated using Eq. 9 with [DBMM] = $1 \mathrm{M}$ for $\mathbf{1}$ and $\mathbf{2}$, and 1 $\mathrm{mM}$ for 4 . ${ }^{c}$ Error bars calculated as twice the standard deviation from three separated measurements. ${ }^{d}$ For PC 4 this was calculated using the previously measured value of $\Phi_{I S C}(0.91$, Table 1$)$. The error bars come from propagating the error of the individual measurements.

\section{Conclusions}

In this work we have measured rate coefficients for dissociative electron transfer $k_{\text {act }}$ for a set of PCs whose structural control through core and $N$-substitution enables manipulation of reactant state electronic structure, spin, and driving force $\left(\Delta G_{E T}^{0}\right)$ in reacting with the alkyl halide initiator DBMM. Additionally, we have quantified reaction yields for the formation of $\mathrm{PC}^{\bullet+}$ photoproducts. From this we draw three sets of interrelated conclusions.

First, we find that the adiabatic Marcus theory of electron transfer is appropriate for modelling the relationship between $k_{a c t}$ and $\Delta G_{E T}^{0}$ for a significant number of systems of varied reactant spin and electronic character. This suggests that the diabatic coupling between reactant 
and product states is large - on the order of $k_{B} T$ - which is likely a manifestation of significant interactions between the $P C \pi^{*}$ system populated in the $S_{1}$ and $T_{1}$ states and antibonding orbitals in the initiator affecting its $\mathrm{C}-\mathrm{Br}$ bond. The efficacy of a general model, with the inference of there being an average electronic coupling relevant for many types of reactant/DBMM combinations, suggests that in these types of systems - i.e., phenoxazines or perhaps more generally chromophores with open and accessible $\pi$ systems - there may be limited value in seeking designs on the sole basis of reactant excited-state electronic structure such as CT versus LE states. Rather the focus should be on driving force, pushing towards diffusion-limited ET behavior, and/or extending the inherent PC lifetimes, which extends the likelihood of encountering initiator while limiting competition from loss pathways. Discussed a different way, it may be inferred that measured photophysical behavior and thermodynamics of new PCs may be sufficient information when coupled with the Marcus curve of Fig. 4, to predict rate constants for DET.

A second set of conclusions follows from the fact that measured $k_{\text {act }}$ values in concert with photophysical knowledge of the PCs allows for modeling of activation (DET) yield as DBMM concentration is varied. Importantly, this can now be visualized as a function of which reactant spin state $\left(S_{1}\right.$ or $\left.T_{1}\right)$ is engaging in the photochemistry. For PC 4, where ISC is high yielding, $T_{1}$ reactivity is dominant at low [DBMM]. However, $S_{1}$ reactivity remains relevant owing to its greater driving force resulting in its larger $k_{\text {act. }}$ Under common synthetic conditions for [DBMM] $\mathrm{S}_{1}$ reactivity accounts for $33 \%$ of DET within an overall yield that is nearly quantitative at $96 \%$. By the same token, systems such as PC 2 that exhibit low ISC yields but significant driving force from their $S_{1}$ state can still be successful photocatalysts, even with short lifetimes. At synthetic [DBMM] the overall activation yield for $\mathbf{2}$ is lessened compared to 4 but it is still $40 \%$, with $80 \%$ of that overall yield coming from the $S_{1}$. Significant $S_{1}$ activation yield improvements are possible for systems with modest increases in lifetime. For example, a PC with the driving force of PC 2 and a 20 ns $S_{1}$ lifetime would yield $75 \%$ driven from the $S_{1}$ alone at the polymerization-relevant initiator concentration. Some caution is warranted regarding exploitation of the $S_{1}$ in O-ATRP. As solution viscosity increases due to polymer growth, diffusion slows, and the advantages of high driving force will be muted with reaction time. Exploitation of primarily $S_{1}$-reacting PCs may need 
to focus on low-viscosity (i.e. common solvents used in synthesis) settings/applications. In photocatalytic cycles where DET is relevant for small-molecule activation and not polymer growth, this will not be an issue. While this study casts PCs 1-4 as reductants for DET, their ample $S_{1}$ and $T_{1}$ excited-state reduction potentials should ensure diffusion-limited ET in systems where bond-breaking does not factor into the reorganization energy cost.

Finally, a third set of conclusions centers on the measurement of photochemical reaction yields as determined by observation of the $\mathrm{PC}^{\bullet+}$ product. This is a significant step beyond treating activation alone because it addresses yields of fragmented DET products, which have successfully avoided potential loss pathways such as back electron transfer (deactivation) following the initial ET event. In other words, even while activation yields are valuable and suggestive, there is no guarantee that forward ET results in the observation of fragmented products. In studies of 4 and 2 under reaction conditions that isolate $T_{1}$ versus $S_{1}$ reactivity, respectively, we observe identical reaction yields of $64 \%$. Factoring spin-state specific loss or formation pathways, it is shown that the $\mathrm{S}_{1}$ of $\mathbf{2}$ and the $\mathrm{T}_{1}$ of $\mathbf{4}$, which possess similar SOMO character, produce $\mathrm{PC}^{\bullet+}$ with an identical $71 \%$ yield. This suggests that reactant spin in these systems does not impact photochemical yield.

There are two reasonable factors contributing to sub-unity $\mathrm{PC}^{\bullet+}$ formation. The first is back electron transfer leading to reformation of the alkyl-halide bond and reduction of PC ${ }^{\bullet+}$. The absence of an observed spin-dependence on the photoreaction quantum yield is not unreasonable as one could imagine there is enough time prior to back electron transfer for dephasing of the spin centers on the alkyl radical and the $\mathrm{PC}^{\bullet+}$, respectively. The second is simple solvent oxidation by $\mathrm{PC}^{\bullet+}$ (or photoexcited $\mathrm{PC}^{\bullet+}$ ) once it is formed by either spin pathway, leading to cation loss and formation of ground-state PC. Such an oxidation would be useful by way of reforming the photocatalyst, but the potential mechanistic role of the nascent solvent radical is unknown and interesting.

Related to this question of solvent oxidation we point to the measured photochemical quantum yield for 1 with a value of $42 \%$ associated with the $S_{1}$ reactant (seen in Table 2). This is notably smaller than what is seen for $\mathbf{2}$ (also an $\mathrm{S}_{1}$ reactor) or $\mathbf{4}$ (a $\mathrm{T}_{1}$ reactor with similar CTbiphenyl character) where a yield of $64-65 \%$ was observed. In the possible limit that solvent oxidation is not a factor, one might be seeing evidence that a CT reactant state ( 2 or $\mathbf{4}$ ) achieves 
a higher reaction yield than non-polar core-delocalized state (1). Since there appears to be no clear mechanistic difference for the forward ET process, this might then indicate that electronic differences can affect loss pathways via back ET, perhaps by way of solvent reorganization effects. However, because $\mathbf{1 c}$ is a stronger oxidant than $\mathbf{2 c}$ or $\mathbf{4 c}$, solvent oxidation may as well be the origin of the yield disparity. Photochemical yield experiments in more oxidatively stable environments are needed to address these issues and experiments along these lines are being considered in ongoing work.

\section{Acknowledgements}

The authors would like to thank Prof. Gordana Dukovic for providing access to her TCSPC instrument, and her graduate students Jesse L. Ruzicka and Dr. Orion M. Pearce for training. Dr. Pearce also participated in many fruitful discussions regarding the interpretation of quenching results. We thank Prof. Josef Michl and his graduate student Andrew Chomas for lending us the mounted LED and driver employed in the measurement of photoreaction quantum yields. This work was supported by the National Science Foundation under Award Number 2016557 and by the National Institutes of Health under Award Number R35GM119702. The content is solely the responsibility of the authors and does not necessarily represent the official views of the National Institutes of Health. SMS and BGM are grateful for support from NSF GRFPs. YML acknowledges support from the Department of Education under a GAANN fellowship. 


\section{References}

1. Kato, M., Kamigaito, M., Sawamoto, M. \& Higashimura, T. Polymerization of Methyl Methacrylate with the Carbon Tetrachloride/Dichlorotris- (triphenylphosphine)ruthenium(II)/Methylaluminum Bis(2,6-di-tert-butylphenoxide) Initiating System: Possibility of Living Radical Polymerization. Macromolecules 28, 1721-1723 (1995).

2. Wang, J.-S. \& Matyjaszewski, K. Controlled/" living" radical polymerization. atom transfer radical polymerization in the presence of transition-metal complexes. J. Am. Chem. Soc. 117, 5614-5615 (1995).

3. Matyjaszewski, K. \& Spanswick, J. Controlled/living radical polymerization. Mater. Today 8, 26-33 (2005).

4. Camenzind, H. et al. Polymers produced by atom transfer radical polymerisation technique with structurally modified terminal groups. (2009).

5. Auschra, C., Eckstein, E., Mühlebach, A., Zink, M.-O. \& Rime, F. Design of new pigment dispersants by controlled radical polymerization. Prog. Org. coatings 45, 83-93 (2002).

6. Bencherif, S. A., Washburn, N. R. \& Matyjaszewski, K. Synthesis by AGET ATRP of degradable nanogel precursors for in situ formation of nanostructured hyaluronic acid hydrogel. Biomacromolecules 10, 2499-2507 (2009).

7. Oh, J. K., Bencherif, S. A. \& Matyjaszewski, K. Atom transfer radical polymerization in inverse miniemulsion: a versatile route toward preparation and functionalization of microgels/nanogels for targeted drug delivery applications. Polymer (Guildf). 50, 4407-4423 (2009).

8. Oh, J. K., Siegwart, D. J. \& Matyjaszewski, K. Synthesis and biodegradation of nanogels as delivery carriers for carbohydrate drugs. Biomacromolecules 8, 3326-3331 (2007).

9. Konkolewicz, D. et al. ICAR ATRP with ppm Cu Catalyst in Water. Macromolecules 45, 4461-4468 (2012).

10. Magenau, A. J. D., Strandwitz, N. C., Gennaro, A. \& Matyjaszewski, K. Electrochemically Mediated Atom Transfer Radical Polymerization. Science (80-. ). 332, 81-84 (2011).

11. Mohapatra, H., Kleiman, M. \& Esser-Kahn, A. P. Mechanically controlled radical polymerization initiated by ultrasound. Nat. Chem. 9, 135 (2017).

12. Kwak, Y. \& Matyjaszewski, K. Photoirradiated Atom Transfer Radical Polymerization with an Alkyl Dithiocarbamate at Ambient Temperature. Macromolecules 43, 5180-5183 (2010).

13. Fors, B. P. \& Hawker, C. J. Control of a Living Radical Polymerization of Methacrylates by Light. Angew. Chemie Int. Ed. 51, 8850-8853 (2012).

14. Mueller, L. \& Matyjaszewski, K. Reducing Copper Concentration in Polymers Prepared via Atom Transfer Radical Polymerization. Macromol. React. Eng. 4, 180-185 (2010).

15. Matyjaszewski, K. Atom Transfer Radical Polymerization (ATRP): Current Status and Future Perspectives. Macromolecules 45, 4015-4039 (2012). 
16. Miyake, G. M. \& Theriot, J. C. Perylene as an Organic Photocatalyst for the Radical Polymerization of Functionalized Vinyl Monomers through Oxidative Quenching with Alkyl Bromides and Visible Light. Macromolecules 47, 8255-8261 (2014).

17. Treat, N. J. et al. Metal-Free Atom Transfer Radical Polymerization. J. Am. Chem. Soc. 136, 1609616101 (2014).

18. Theriot, J. C. et al. Organocatalyzed atom transfer radical polymerization driven by visible light. Science (80-. ). 352, 1082 LP - 1086 (2016).

19. Cole, J. P., Federico, C. R., Lim, C.-H. \& Miyake, G. M. Photoinduced organocatalyzed atom transfer radical polymerization using low ppm catalyst loading. Macromolecules 52, 747-754 (2019).

20. Du, Y. et al. Strongly Reducing, Visible-Light Organic Photoredox Catalysts as Sustainable Alternatives to Precious Metals. Chem. - A Eur. J. 23, 10962-10968 (2017).

21. Pearson, R. M., Lim, C. H., McCarthy, B. G., Musgrave, C. B. \& Miyake, G. M. Organocatalyzed Atom Transfer Radical Polymerization Using N-Aryl Phenoxazines as Photoredox Catalysts. J. Am. Chem. Soc. 138, 11399-11407 (2016).

22. McCarthy, B. G. et al. Structure-Property Relationships for Tailoring Phenoxazines as Reducing Photoredox Catalysts. J. Am. Chem. Soc. 140, 5088-5101 (2018).

23. Park, G. S., Back, J., Choi, E. M., Lee, E. \& Son, K. Visible light-mediated metal-free atom transfer radical polymerization with $\mathrm{N}$-trifluoromethylphenyl phenoxazines. Eur. Polym. J. 117, 347-352 (2019).

24. Discekici, E. H., Anastasaki, A., Read de Alaniz, J. \& Hawker, C. J. Evolution and future directions of metal-free atom transfer radical polymerization. Macromolecules 51, 7421-7434 (2018).

25. Dadashi-Silab, S., Pan, X. \& Matyjaszewski, K. Phenyl Benzo[b]phenothiazine as a Visible Light Photoredox Catalyst for Metal-Free Atom Transfer Radical Polymerization. Chem. - A Eur. J. 23, 5972-5977 (2017).

26. Corbin, D. A., Lim, C.-H. \& Miyake, G. M. Phenothiazines, Dihydrophenazines, and Phenoxazines: Sustainable Alternatives to Precious-Metal-Based Photoredox Catalysts. Aldrichimica Acta 52, 7 (2019).

27. Buss, B. L., Lim, C.-H. \& Miyake, G. M. Dimethyl Dihydroacridines as Photocatalysts in Organocatalyzed Atom Transfer Radical Polymerization of Acrylate Monomers. Angew. Chemie Int. Ed. 59, 3209-3217 (2020).

28. Huang, Z. et al. Metal-Free Atom Transfer Radical Polymerization of Methyl Methacrylate with ppm Level of Organic Photocatalyst. Macromol. Rapid Commun. 38, 1600461 (2017).

29. Kutahya, C. et al. Photoinduced Metal-Free Atom Transfer Radical Polymerization Using Highly Conjugated Thienothiophene Derivatives. Macromolecules 50, 6903-6910 (2017).

30. Corrigan, N., Yeow, J., Judzewitsch, P., Xu, J. \& Boyer, C. Seeing the Light: Advancing Materials Chemistry through Photopolymerization. Angew. Chemie Int. Ed. 58, 5170-5189 (2019).

31. Jung, K. et al. Designing with Light: Advanced 2D, 3D, and 4D Materials. Adv. Mater. 32, 1903850 (2020). 
32. Corrigan, N., Shanmugam, S., Xu, J. \& Boyer, C. Photocatalysis in organic and polymer synthesis. Chem. Soc. Rev. 45, 6165-6212 (2016).

33. Lee, Y. \& Kwon, M. S. Emerging Organic Photoredox Catalysts for Organic Transformations. European J. Org. Chem. 2020, 6028-6043 (2020).

34. Singh, V. K. et al. Highly efficient organic photocatalysts discovered via a computer-aided-design strategy for visible-light-driven atom transfer radical polymerization. Nat. Catal. 1, 794-804 (2018).

35. Pan, X. et al. Mechanism of Photoinduced Metal-Free Atom Transfer Radical Polymerization: Experimental and Computational Studies. J. Am. Chem. Soc. 138, 2411-2425 (2016).

36. Tang, W. \& Matyjaszewski, K. Effects of Initiator Structure on Activation Rate Constants in ATRP. Macromolecules 40, 1858-1863 (2007).

37. Horn, M. \& Matyjaszewski, K. Solvent effects on the activation rate constant in atom transfer radical polymerization. Macromolecules 46, 3350-3357 (2013).

38. Gillies, M. B. et al. A DFT Study of R-X Bond Dissociation Enthalpies of Relevance to the Initiation Process of Atom Transfer Radical Polymerization. Macromolecules 36, 8551-8559 (2003).

39. Koyama, D., Dale, H. J. A. \& Orr-Ewing, A. J. Ultrafast Observation of a Photoredox Reaction Mechanism: Photoinitiation in Organocatalyzed Atom-Transfer Radical Polymerization. J. Am. Chem. Soc. 140, 1285-1293 (2018).

40. Jockusch, S. \& Yagci, Y. The active role of excited states of phenothiazines in photoinduced metal free atom transfer radical polymerization: singlet or triplet excited states? Polym. Chem. 7, 60396043 (2016).

41. Turro, N. J. Modern Molecular Photochemistry. (University Science Books, 1991).

42. Sartor, S. M., McCarthy, B. G., Pearson, R. M., Miyake, G. M. \& Damrauer, N. H. Exploiting Charge-Transfer States for Maximizing Intersystem Crossing Yields in Organic Photoredox Catalysts. J. Am. Chem. Soc. 140, 4778-4781 (2018).

43. Sartor, S. M., Lattke, Y. M., McCarthy, B. G., Miyake, G. M. \& Damrauer, N. H. Effects of Naphthyl Connectivity on the Photophysics of Compact Organic Charge-Transfer Photoredox Catalysts. J. Phys. Chem. A 123, 4727-4736 (2019).

44. van Willigen, H., Jones, G. \& Farahat, M. S. Time-resolved EPR study of photoexcited triplet-state formation in electron-donor-substituted acridinium ions. J. Phys. Chem. 100, 3312-3316 (1996).

45. Okada, T. et al. Ultrafast intersystem crossing in some intramolecular heteroexcimers. J. Phys. Chem. 85, 3957-3960 (1981).

46. Kim, H. \& Scholes, G. D. Configuration mixing upon reorganization of dihedral angle induces rapid intersystem crossing in organic photoredox catalyst. Phys. Chem. Chem. Phys. 22, 13292-13298 (2020).

47. Lim, C.-H. et al. Intramolecular Charge Transfer and lon Pairing in N,N-Diaryl Dihydrophenazine Photoredox Catalysts for Efficient Organocatalyzed Atom Transfer Radical Polymerization. J. Am. Chem. Soc. 139, 348-355 (2017). 
48. Lakowicz, J. R. Principles of Fluorescence Spectroscopy. (2013).

49. Eberson, L. Electron Transfers in Organic Chemistry. (Springer-Verlag, 1987).

50. Kavarnos, G. J. Fundamentals of Photoinduced Electron Transfer. (VCH, 1993).

51. Cannon, R. D. Electron Transfer Reactions. (Butterworth, 1980).

52. Arceo, E., Montroni, E. \& Melchiorre, P. Photo-Organocatalysis of Atom-Transfer Radical Additions to Alkenes. Angew. Chemie Int. Ed. 53, 12064-12068 (2014).

53. Luo, Y.-R. Handbook of bond dissociation energies in organic compounds. (CRC press, 2002).

54. Nain, A. K. Densities, ultrasonic speeds, viscosities and excess properties of binary mixtures of methyl methacrylate with $\mathrm{N}, \mathrm{N}$-dimethylformamide and $\mathrm{N}, \mathrm{N}$-dimethylacetamide at different temperatures. J. Chem. Thermodyn. 60, 105-116 (2013).

55. Eberson, L. Electron transfer reactions in organic chemistry. II. An analysis of alkyl halide reduction by electron transfer reagents on the basis of the Marcus theory. Acta Chem. Scand. $B$ 36, 533-543 (1982).

56. Saveant, J. M. A simple model for the kinetics of dissociative electron transfer in polar solvents. Application to the homogeneous and heterogeneous reduction of alkyl halides. J. Am. Chem. Soc. 109, 6788-6795 (1987).

57. Andrieux, C. P., Le Gorande, A. \& Saveant, J. M. Electron transfer and bond breaking. Examples of passage from a sequential to a concerted mechanism in the electrochemical reductive cleavage of arylmethyl halides. J. Am. Chem. Soc. 114, 6892-6904 (1992).

58. Saveant, J. M. Electron transfer, bond breaking, and bond formation. Acc. Chem. Res. 26, 455461 (1993).

59. Rehm, D. \& Weller, A. Kinetics of Fluorescence Quenching by Electron and H-Atom Transfer. Isr. J. Chem. 8, 259-271 (1970).

60. Pause, L., Robert, M. \& Savéant, J. Quantum Yields Lower than Unity in Photo-induced Dissociative Electron Transfers: The Reductive Cleavage of Carbon Tetrachloride. ChemPhysChem 1, 199-205 (2000).

61. Turró, C., Zaleski, J. M., Karabatsos, Y. M. \& Nocera, D. G. Bimolecular Electron Transfer in the Marcus Inverted Region. J. Am. Chem. Soc. 118, 6060-6067 (1996).

62. Murov, S. L., Carmichael, I. \& Hug, G. Handbook of Photochemistry. in 298-304 (Marcel Dekker, Inc., 1993).

63. Zepp, R. G. Quantum yields for reaction of pollutants in dilute aqueous solution. Environ. Sci. Technol. 12, 327-329 (1978).

64. Zepp, R. G. \& Cline, D. M. Rates of direct photolysis in aquatic environment. Environ. Sci. Technol. 11, 359-366 (1977). 


\section{For Table of Contents Only}

Classical Marcus-Savéant Theory

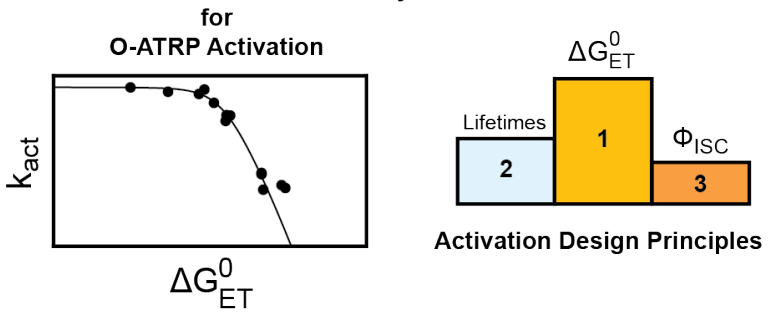

